\title{
Multispecies Weighted Hurwitz Numbers ${ }^{\star}$
}

\author{
J. HARNAD †‡ \\ † Centre de recherches mathématiques, Université de Montréal, \\ C.P. 6128, succ. Centre-ville, Montréal (QC) H3C 3J\%, Canada \\ E-mail: harnad@crm.umontreal.ca \\ $\ddagger$ Department of Mathematics and Statistics, Concordia University, \\ 7141 Sherbrooke W., Montréal (QC) H4B 1R6, Canada
}

Received March 31, 2015, in final form November 16, 2015; Published online December 02, 2015 http://dx.doi.org/10.3842/SIGMA.2015.097

\begin{abstract}
The construction of hypergeometric $2 D$ Toda $\tau$-functions as generating functions for weighted Hurwitz numbers is extended to multispecies families. Both the enumerative geometrical significance of multispecies weighted Hurwitz numbers, as weighted enumerations of branched coverings of the Riemann sphere, and their combinatorial significance in terms of weighted paths in the Cayley graph of $S_{n}$ are derived. The particular case of multispecies quantum weighted Hurwitz numbers is studied in detail.
\end{abstract}

Key words: weighted Hurwitz number; $\tau$-function; multispecies

2010 Mathematics Subject Classification: 05A15; 14H30; 33C70; 57M12

\section{Introduction}

In $[11,12]$ a simple method was developed for constructing parametric families of $\mathrm{KP}$ and $2 D$ Toda $\tau$-functions $[28,29,30]$ of hypergeometric type $[10,25]$ that serve as generating functions for the weighted enumeration of $n$-sheeted branched coverings of the Riemann sphere. These are characterized by the fact that their expansions in the basis of products of Schur functions have only diagonal coefficients, and these are of special content product form. When expanded instead in the basis of products of power sum symmetric functions, the coefficients turn out to be weighted sums of Hurwitz numbers, with weighting dependent generally on an infinite sequence of parameters $\mathbf{c}=\left(c_{1}, c_{2}, \ldots\right)$ determined by an associated weight generating function $G(z)$. Such weighted sums may be interpreted equivalently as weighted enumeration of paths in the Cayley graph of the symmetric group $S_{n}$ generated by transpositions.

The special choice $G(z)=\exp (z)$ gives rise to the generating functions for simple and double Hurwitz numbers introduced by Pandharipande [26] and Okounkov [24], in which all branch points other than a single one or a specified pair have simple branching profiles. Further insight into the significance of these hypergeometric $\tau$-functions as generating functions in terms of recurrence relations for triangulations was developed in [9]. More generally, the "topological recursion" program was successfully applied to the study of Hurwitz numbers in $[1,3,4,5]$.

It has been known since the pioneering works of Hurwitz [7, 8], Frobenius [16, 17] and Schur [27] that these numbers may be reinterpreted combinatorially using the monodromy representation of the fundamental group of the punctured sphere with values in $S_{n}$ determined by lifting closed paths to the covering surface. From this viewpoint, they enumerate factorizations of the identity element into products of elements whose conjugacy classes correspond to the ramifications profiles, and hence give uniformly weighted enumeration of paths in the Cayley graph from one conjugacy class to another with a given number of steps.

\footnotetext{
${ }^{\star}$ This paper is a contribution to the Special Issue on Exact Solvability and Symmetry Avatars in honour of Luc Vinet. The full collection is available at http://www.emis.de/journals/SIGMA/ESSA2014.html
} 
Another choice of weight generating function that was studied in [12] is the quantum dilogarithm function [6]. This amounts to equating the parameters $c_{i}$ to powers of a single quantum deformation parameter $q$, and gives rise to four special versions of quantum weighted Hurwitz numbers, whose distribution functions were linked to those of a Bosonic gas with linear energy spectrum in [12]. Using a suitably extended class of weight generating functions, this was further generalized in $[13,14]$ to include both the infinite family of classical weighting parameters $\mathbf{c}$ and the pair $(q, t)$ of quantum deformation parameters characterizing Macdonald polynomials.

In the present work, the notion of weighted Hurwitz numbers is extended to weighted enumerations of multispecies coverings involving arbitrary choices for the corresponding weighting parameters. The generating function depends on $l+m$ expansion parameters $\left(w_{1}, \ldots, w_{l} ; z_{1}, \ldots, z_{m}\right)$, corresponding to two classes and $l+m$ subspecies (or "colours") of branch points with ramification profiles types $\left\{\mu^{(\beta)}\right\}_{\beta=1, \ldots, l}$ and $\left\{\nu^{(\beta)}\right\}_{\beta=1, \ldots, m}$. The special case of signed multispecies enumeration in the uniformly weighted case was studied in [15]. Its combinatorial significance was explained in terms of enumeration of paths in the Cayley graph that are subdivided into strictly or weakly monotonically increasing subsequences of transpositions having given lengths. In the single species case [12], this was shown to be equivalent to (signed) enumeration of branched covers of the Riemann sphere with the "coloured" branch points constrained to have fixed total ramification index within each class. Another special case detailed here consists of "multispecies quantum weighted Hurwitz numbers", in which the weighting parameters consist of powers of a sequence of auxiliary quantum deformation parameters $\mathbf{q}=\left(q_{1}, \ldots, q_{l}\right), \mathbf{p}=$ $\left(p_{1}, \ldots, p_{m}\right)$.

In Sections 1.1-1.4 the basic notions regarding Hurwitz numbers will be recalled, together with the construction of weighted Hurwitz numbers using infinite parameter families of weight generating functions $G(z)$, as developed in $[11,12,15]$. In Section 2 , the single species case will be extended to multispecies by introducing the idea of "coloured" branch points, of two classes. Weight generating functions depending on a multiparametric set of expansion parameters multiplicatively provide multiparametric families of $2 D$ Toda $\tau$-functions of hypergeometric type that are generating functions for such multispecies weighted Hurwitz numbers. As in the single species case, these may be viewed both geometrically and combinatorially, in terms of weighted coverings and paths in the Cayley graph. In Section 3 this is restricted to the special cases of quantum weightings introduced in [12] to produce generating functions for multispecies quantum Hurwitz numbers. These are interpreted, as in the single species case, both geometrically and combinatorially ${ }^{1}$.

\subsection{Hurwitz numbers}

For a set of $k \in \mathbf{N}^{+}$partitons $\left(\mu^{(1)}, \ldots, \mu^{(k)}\right)$ of $n \in \mathbf{N}^{+}$, let $H\left(\mu^{(1)}, \ldots, \mu^{(k)}\right)$ denote the number of $n$-sheeted branched coverings of the Riemann sphere (not necessarily connected), with $k$ branch points, whose ramification profiles are given by the partitions, weighted by the inverse of the order of the automorphism group. These are the geometrically defined Hurwitz numbers as originally studied by Hurwitz [16, 17, 19]. The genus $g$ of the covering surface is determined by the Riemann Hurwitz formula for the Euler characteristic

$$
2-2 g=2 n-\sum_{i=1}^{k} \ell^{*}\left(\mu^{(i)}\right),
$$

where

$$
\ell^{*}(\mu):=|\mu|-\ell(\mu)
$$

\footnotetext{
${ }^{1}$ A different multiparametric family of generating functions for weighted Hurwitz numbers over $\mathbf{R} \mathbf{P}^{2}$, consisting of BKP generating functions of hypergeometric type, was considered in [22, 23].
} 
is the colength of the partition $\mu$, i.e., the complement of the length $\ell(\mu)$ with respect to its weight $|\mu|$, or the degree of degeneracy of the branched cover over a point with ramification profile type $\mu$.

The Frobenius-Schur formula $[7,8,19,27]$ expresses these as sums over irreducible $S_{n}$ characters

$$
H\left(\mu^{(1)}, \ldots, \mu^{(k)}\right)=\sum_{\lambda,|\lambda|=n} h_{\lambda}^{k-2} \prod_{i=1}^{k} \frac{\chi_{\lambda}\left(\mu^{(i)}\right)}{z_{\mu^{(i)}}},
$$

where $\chi_{\lambda}(\mu)$ is the character of the irreducible representation of symmetry type $\lambda$, evaluated on the conjugacy class $\operatorname{cyc}(\mu)$ consisting of elements with cycle lengths equal to the parts of the partition $\mu$,

$$
h_{\lambda}=\operatorname{det}\left(\frac{1}{\left(\lambda_{i}-i+j\right) !}\right)^{-1}
$$

is the product of the hook lengths in the Young diagram of partition $\lambda$ and

$$
z_{\mu}=\prod_{i \in \mathbf{N}} i^{m_{i}(\mu)}\left(m_{i}(\mu)\right) !
$$

is the order of the stabilizer under conjugation of any element of the conjugacy class cyc $(\mu)$, with $m_{i}(\mu)$ the number of parts of the partition $\mu$ equal to $i$,

There is an alternative interpretation of $H\left(\mu^{(1)}, \ldots, \mu^{(k)}\right)$ that is purely combinatorial; it equals $\frac{1}{n !}$ times the number of ways in which the identity element $\mathbf{I} \in S_{n}$ may be expressed as a product of $k$ elements belonging to the conjugacy classes of cycle type $\left\{\operatorname{cyc}\left(\mu^{(i)}\right)\right\}_{i=1, \ldots, k}$

$$
\mathbf{I}=g_{1} g_{2} \cdots g_{k}, \quad \text { where } \quad g_{i} \in \operatorname{cyc}\left(\mu^{(i)}\right) .
$$

The two are related by noting that each such factorization may be understood as defining the image in $S_{n}$ of the identity element in the fundamental group of the punctured sphere with the branch points removed under the monodromy map obtained by lifting closed loops to the covering surface.

\subsection{Weighted geometrical Hurwitz numbers}

As defined in [12], given a weight generating function $G(z)$ expressible as an infinite product

$$
G(z)=\prod_{i=1}^{\infty}\left(1+c_{i} z\right), \quad \mathbf{c}=\left(c_{1}, c_{2}, \ldots\right),
$$

the weight $W_{G}\left(\mu^{(1)}, \ldots, \mu^{(k)}\right)$ assigned to a configuration of $k+2$ branch points with ramification profiles $\left\{\mu^{(1)}, \ldots, \mu^{(k)}, \mu, \nu\right\}$ is solely determined by the colengths $\left\{\ell^{*}\left(\mu^{(1)}\right), \ldots, \ell^{*}\left(\mu^{(k)}\right)\right\}$, and is given by evaluation of the monomial sum symmetric functions at the parameter values

$$
W_{G}\left(\mu^{(1)}, \ldots, \mu^{(k)}\right):=m_{\lambda}(\mathbf{c})=\frac{1}{|\operatorname{aut}(\lambda)|} \sum_{\sigma \in S_{k}} \sum_{1 \leq i_{1}<\cdots<i_{k}} c_{i_{\sigma(1)} \ell^{*}\left(\mu^{(1)}\right)} \cdots c_{i_{\sigma(k)}}^{\ell^{*}\left(\mu^{(k)}\right)} .
$$

Here $\lambda$ is the partition of length $\ell(\lambda)=k$ whose parts are $\left\{\ell^{*}\left(\mu^{(i)}\right)\right\}_{i=1, \ldots, k}$, and $|\operatorname{aut}(\lambda)|$ is the order of the automorphism group of $\lambda$

$$
|\operatorname{aut}(\lambda)|:=\prod_{i=1}^{\ell(\lambda}\left(m_{i}(\lambda)\right) !
$$

where $m_{i}(\lambda)$ is the number of parts of $\lambda$ equal to $i$. 
The geometrically defined double weighted Hurwitz numbers $H_{G}^{d}(\mu, \nu)$ give a weighted enumeration of $n$-sheeted branched covers of the Riemann sphere that contain a pair of fixed branch points, say at $(0, \infty)$, with ramification profile types given by the pair of partitions $(\mu, \nu)$ and a further set of $k$ branch points with ramification profiles $\left(\mu^{(1)}, \ldots, \mu^{(k)}\right)$. They are defined by the weighted sums

$$
H_{G}^{d}(\mu, \nu):=\sum_{k=0}^{\infty} \sum_{\substack{\mu^{(1)}, \ldots, \mu^{(k)} \\ \sum_{i=1}^{k} \ell^{*}\left(\mu^{(i)}\right)=d}}^{\prime} W_{G}\left(\mu^{(1)}, \ldots, \mu^{(k)}\right) H\left(\mu^{(1)}, \ldots, \mu^{(k)}, \mu, \nu\right),
$$

over all $k$-tuples of nontrivial ramification profiles satisfying the condition

$$
d=\sum_{i=1}^{k} \ell^{*}\left(\mu^{(i)}\right)=|\lambda|
$$

with weight $W_{G}\left(\mu^{(1)}, \ldots, \mu^{(k)}\right)$ given by (1.8).

The Riemann-Hurwitz formula for the genus $g$ of the covering surface is then

$$
2-2 g=\ell(\mu)+\ell(\nu)-d .
$$

An alternative is to use the dual weight generating function

$$
\tilde{G}(z)=\prod_{i=1}^{\infty}\left(1-\tilde{c}_{i} z\right)^{-1}, \quad \tilde{\mathbf{c}}=\left(\tilde{c}_{1}, \tilde{c}_{2}, \ldots\right),
$$

for which the geometrical weight $W_{\tilde{G}}\left(\mu^{(1)}, \ldots, \mu^{(k)}\right)$ is given by the "forgotten" symmetric function $f_{\lambda}(\tilde{\mathbf{c}}), \tilde{\mathbf{c}}=\left(\tilde{c}_{1}, \tilde{c}_{2}, \ldots\right)$

$$
W_{\tilde{G}}\left(\mu^{(1)}, \ldots, \mu^{(k)}\right):=f_{\lambda}(\tilde{\mathbf{c}})=\frac{(-1)^{\ell^{*}(\lambda)}}{|\operatorname{aut}(\lambda)|} \sum_{\sigma \in S_{k}} \sum_{1 \leq i_{1} \leq \cdots \leq i_{k}} \tilde{c}_{i_{\sigma(1)}}^{\ell^{*}\left(\mu^{(1)}\right)} \cdots \tilde{c}_{i_{\sigma(k)}}^{\ell^{*}\left(\mu^{(k)}\right)},
$$

where the partition $\lambda$ is again defined as above, with parts consisting of the colengths $\left\{\ell^{*}\left(\mu^{(i)}\right)\right\}_{i=1, \ldots, k}$. The dually weighted geometrical Hurwitz numbers are similarly defined by the weighted sum

$$
H_{\tilde{G}}^{d}(\mu, \nu):=\sum_{k=0}^{\infty} \sum_{\substack{\mu^{(1)}, \ldots, \mu^{(k)} \\ \sum_{i=1}^{k} \ell^{*}\left(\mu^{(i)}\right)=d}}^{\prime} W_{\tilde{G}}\left(\mu^{(1)}, \ldots, \mu^{(k)}\right) H\left(\mu^{(1)}, \ldots, \mu^{(k)}, \mu, \nu\right) .
$$

\subsection{Weighted combinatorial Hurwitz numbers}

Following [11, 12], we may alternatively define a combinatorial Hurwitz number $F_{G}^{d}(\mu, \nu)$ that gives the weighted enumeration of $d$-step paths in the Cayley graph of the symmetric group $S_{n}$ generated by transpositions $(a, b), b>a$, starting at an element $h \in \operatorname{cyc}(\mu)$ in the conjugacy class $\operatorname{cyc}(\mu)$ consisting of elements with cycle lengths equal to the parts of $\mu$ and ending in the conjugacy class $\operatorname{cyc}(\nu)$

$$
\left(a_{d} b_{d}\right) \cdots\left(a_{1} b_{1}\right) h \in \operatorname{cyc}(\nu) .
$$

Every such path has a signature $\lambda$, which is defined to be the partition of weight $d$, whose parts are, in weakly decreasing order, the number of times any given second element $b_{i}, i=1, \ldots, \ell(\lambda)$ 
is repeated. In the case of the weight generating function $G(z)$, we assign to any path with signature $\lambda$ a combinatorial weight equal to the product $e_{\lambda}(\mathbf{c})$ of the elementary symmetric functions [20], evaluated at the parameters $\left(c_{a}, c_{2}, \ldots\right)$

$$
e_{\lambda}(\mathbf{c})=\prod_{i=1}^{\ell(\lambda)} e_{\lambda_{i}}(\mathbf{c})
$$

In the case of the dual generating functions $\tilde{G}(z)$, we assign a combinatorial weight equal to the product $h_{\lambda}(\mathbf{c})$ of the complete symmetric functions [20], evaluated at the parameters $\left(\tilde{c}_{1}, \tilde{c}_{2}, \ldots\right)$

$$
h_{\lambda}(\tilde{\mathbf{c}})=\prod_{i=1}^{\ell(\lambda)} h_{\lambda_{i}}(\tilde{\mathbf{c}})
$$

Let $m_{\mu \nu}^{\lambda}$ be the number of $d=|\lambda|$ step paths of signature $\lambda$ starting at $h \in \operatorname{cyc}(\mu)$ and ending in the conjugacy class $\operatorname{cyc}(\nu)$. Then the combinatorial weighted Hurwitz numbers $F_{G}^{d}(\mu, \nu)$, $F_{\tilde{G}}^{d}(\mu, \nu)$ are defined to be the weighted sums

$$
\begin{aligned}
& F_{G}^{d}(\mu, \nu):=\frac{1}{n !} \sum_{\lambda} e_{\lambda}(\mathbf{c}) m_{\mu \nu}^{\lambda}, \\
& F_{\tilde{G}}^{d}(\mu, \nu):=\frac{1}{n !} \sum_{\lambda} h_{\lambda}(\tilde{\mathbf{c}}) m_{\mu \nu}^{\lambda} .
\end{aligned}
$$

In [12] it is proved that these two notions of weighted Hurwitz numbers in fact coincide:

Theorem 1.1 ([12]).

$$
F_{G}^{d}(\mu, \nu)=H_{G}^{d}(\mu, \nu), \quad F_{\tilde{G}}^{d}(\mu, \nu)=H_{\tilde{G}}^{d}(\mu, \nu) .
$$

The main idea behind the proof is to define associated elements $G_{n}(w, \mathcal{J}) \in \mathbf{Z}\left(\mathbf{C}\left[S_{n}\right]\right)$ and $\tilde{G}_{n}(z, \mathcal{J}) \in \mathbf{Z}\left(\mathbf{C}\left[S_{n}\right]\right)$ in the centre $\mathbf{Z}\left(\mathbf{C}\left[S_{n}\right]\right)$ of the group algebra $\mathbf{C}\left[S_{n}\right]$ by

$$
G_{n}(z, \mathcal{J}):=\prod_{a=1}^{n} G\left(z \mathcal{J}_{a}\right), \quad \tilde{G}(z, \mathcal{J}):=\prod_{a=1}^{n} \tilde{G}\left(z \mathcal{J}_{a}\right),
$$

where $\mathcal{J}:=\left(\mathcal{J}_{1}, \ldots, \mathcal{J}_{n}\right)$ are the Jucys-Murphy elements $[2,18,21]$

$$
\mathcal{J}_{1}:=0, \quad \mathcal{J}_{b}:=\sum_{a=1}^{b-1}(a b), \quad b=1, \ldots, n,
$$

which generate an abelian subalgebra of the centre $\mathbf{Z}\left(\mathbf{C}\left[S_{n}\right]\right)$ of the group algebra $\mathbf{C}\left[S_{n}\right]$.

The elements $G_{n}(z, \mathcal{J})$ and $\tilde{G}_{n}(z, \mathcal{J})$ define endomorphisms of $\mathbf{Z}\left(\mathbf{C}\left[S_{n}\right]\right)$ under multiplication, which are diagonal in the basis $\left\{F_{\lambda}\right\}$ of $\mathbf{Z}\left(\mathbf{C}\left[S_{n}\right]\right)$ consisting of the orthogonal idempotents, corresponding to irreducible representations, labelled by partitions $\lambda$ of $n$

$$
G_{n}(z, \mathcal{J}) F_{\lambda}=r_{\lambda}^{G(z)} F_{\lambda}, \quad \tilde{G}_{n}(z, \mathcal{J}) F_{\lambda}=r_{\lambda}^{\tilde{G}(z)} F_{\lambda}
$$

with eigenvalues of the following content product form

$$
r_{\lambda}^{G(z)}(N):=\prod_{(i, j) \in \lambda} G(z(N+j-i)), \quad r_{\lambda}^{\tilde{G}(z)}(N):=\prod_{(i, j) \in \lambda} \tilde{G}(z(N+j-i)) .
$$


On the other hand, the Cauchy-Littlewood generating function relation [20] and its dual show that $G_{n}(z, \mathcal{J})$ and $\tilde{G}_{n}(z, \mathcal{J})$ may be expanded in terms of dual bases of the algebra of symmetric functions, evaluated either on the parameters $\mathbf{c}$ or on the Jucys-Murphy elements $\mathcal{J}=\left(\mathcal{J}_{1}, \ldots, \mathcal{J}_{n}\right)$

$$
\begin{aligned}
G_{n}(z, \mathcal{J}) & =\sum_{\lambda,|\lambda|=n} e_{\lambda}(\mathbf{c}) m_{\lambda}(\mathcal{J})=\sum_{\lambda,|\lambda|=n} m_{\lambda}(\mathbf{c}) e_{\lambda}(\mathcal{J}), \\
\tilde{G}_{n}(z, \mathcal{J}) & =\sum_{\lambda,|\lambda|=n} h_{\lambda}(\mathbf{c}) m_{\lambda}(\mathcal{J})=\sum_{\lambda,|\lambda|=n} f_{\lambda}(\mathbf{c}) e_{\lambda}(\mathcal{J}),
\end{aligned}
$$

where $e_{\lambda}, h_{\lambda}, m_{\lambda}$ and $f_{\lambda}$ are the elementary, complete, monomial and "forgotten" symmetric functions [20], respectively. Applying (1.26) and (1.27) to the basis for $\mathbf{Z}\left(\mathbf{C}\left[S_{n}\right]\right)$ consisting of the cycle sums

$$
C_{\mu}:=\sum_{h \in \operatorname{cyc}(\mu)} h
$$

and using the identities

$$
e_{j}(\mathcal{J})=\sum_{\mu, \ell^{*}(\mu)=j} C_{\mu} \quad \text { and } \quad m_{\lambda}(\mathcal{J}) C_{\mu}=\sum_{\nu,|\nu|=|\mu|} m_{\mu \nu}^{\lambda} C_{\nu}
$$

leads to (1.21).

The bases $\left\{F_{\lambda}\right\}_{|\lambda|=n}$ and $\left\{C_{\mu}\right\}_{|\mu|=n}$ are related by

$$
F_{\lambda}=h_{\lambda} \sum_{\mu,|\mu|=|\lambda|} \chi_{\lambda}(\mu) C_{\mu}
$$

where $\chi_{\lambda}(\mu)$ denotes the irreducible character of the irreducible representation of type $\lambda$ evaluated on the conjugacy class $\operatorname{cyc}(\mu)$. Under the characteristic map, this is equivalent to the Frobenius character formula [20]

$$
s_{\lambda}=\sum_{\mu,|\mu|=|\lambda|} z_{\mu}^{-1} \chi_{\lambda}(\mu) p_{\mu}
$$

\subsection{Hypergeometric $2 D$ Toda $\tau$-functions as generating functions}

As shown in [12], for any given generating function of type $G(z)$ or $\tilde{G}(z)$, there is a naturally associated $2 D$ Toda $\tau$-function of hypergeometric type, expressible as a diagonal double Schur function expansion

$$
\begin{aligned}
\tau^{G(z)}(N, \mathbf{t}, \mathbf{s}) & :=\sum_{\lambda} r_{\lambda}^{G(z)}(N) s_{\lambda}(\mathbf{t}) s_{\lambda}(\mathbf{s}), \\
\tau^{\tilde{G}(z)}(N, \mathbf{t}, \mathbf{s}) & :=\sum_{\lambda} r_{\lambda}^{\tilde{G}(z)}(N) s_{\lambda}(\mathbf{t}) s_{\lambda}(\mathbf{s}),
\end{aligned}
$$

where

$$
\mathbf{t}=\left(t_{1}, t_{2}, \ldots\right), \quad \mathbf{s}=\left(s_{1}, s_{2}, \ldots\right)
$$

are the $2 D$ Toda flow variables, which may be identified in this notation with the power sums

$$
t_{i}=\frac{p_{i}}{i}, \quad s_{i}=\frac{p_{i}^{\prime}}{i}, \quad N \in \mathbf{Z}
$$


in two independent sets of variables. (See [20] for notation and further definitions involving symmetric functions.) The coefficients have the standard content product form that characterize such $2 D \tau$-functions of hypergeometric kind

$$
r_{\lambda}^{G(z)}(N):=r_{0}^{G(z)}(N) \prod_{(i, j) \in \lambda} G(z(N+j-i)),
$$

where

$$
\begin{aligned}
& r_{0}^{G(z)}(N):=\prod_{j=1}^{N-1} G((N-j) z)^{j}, \quad r_{0}^{G(z)}(0):=1, \\
& r_{0}^{G(z)}(-N):=\prod_{j=1}^{N} G((j-N) z)^{-j}, \quad N \geq 1,
\end{aligned}
$$

and identical formulae for $G$ replaced by $\tilde{G}$.

The other main result of [12] is that the resulting $\tau$-functions (1.32), (1.33), for $N=0$

$$
\tau^{G(z)}(\mathbf{t}, \mathbf{s}):=\tau^{G(z)}(0, \mathbf{t}, \mathbf{s}), \quad \tau^{\tilde{G}(z)}(\mathbf{t}, \mathbf{s}):=\tau^{\tilde{G}(z)}(0, \mathbf{t}, \mathbf{s}),
$$

when expanded in the basis of tensor products of pairs of power sum symmetric functions $\left\{p_{\mu}\right\}$, using the Frobenius character formula (1.31), are generating functions for the weighted double Hurwitz numbers.

Theorem $1.2([12])$.

$$
\begin{aligned}
& \tau^{G(z)}(\mathbf{t}, \mathbf{s})=\sum_{d=0}^{\infty} \sum_{\substack{\mu, \nu,|\mu|=|\nu|}} z^{d} H_{G}^{d}(\mu, \nu) p_{\mu}(\mathbf{t}) p_{\nu}(\mathbf{s}), \\
& \tau^{\tilde{G}(z)}(\mathbf{t}, \mathbf{s})=\sum_{d=0}^{\infty} \sum_{\substack{\mu, \nu,|\mu|=|\nu|}} z^{d} H_{\tilde{G}}^{d}(\mu, \nu) p_{\mu}(\mathbf{t}) p_{\nu}(\mathbf{s}) .
\end{aligned}
$$

\section{$2 \quad 2 D$ Toda $\tau$-functions as generating functions for multispecies weighted Hurwitz numbers}

For any choice of weight generating functions $G^{1}\left(w_{1}\right), G^{2}\left(w_{2}\right), \ldots, \tilde{G}^{1}\left(z_{1}\right), \tilde{G}^{2}\left(z_{2}\right), \ldots$, we may form composites by using the product $\prod_{\alpha} G^{\alpha}\left(w_{\alpha}\right) \prod_{\beta} \tilde{G}^{\beta}\left(z_{\beta}\right)$ as generating function for multiple weighting types. The resulting content product coefficients $r_{\lambda}^{\prod_{\alpha} G^{\alpha}\left(w_{\alpha}\right) \prod_{\beta} \tilde{G}^{\beta}\left(w_{\beta}\right.}$ are just the product $\prod_{\alpha} r_{\lambda}^{G^{\alpha}\left(w_{\alpha}\right)} \prod_{\beta} r_{\lambda}^{\tilde{G}^{\beta}\left(z_{\beta}\right)}$ of the individual ones

$$
r_{\lambda}^{G^{\alpha}\left(w_{\alpha}\right)}:=\prod_{(i, j) \in \lambda} G^{\alpha}\left(w_{\alpha}(N+j-i)\right), \quad r_{\lambda}^{\tilde{G}^{\beta}\left(z_{\beta}\right)}:=\prod_{(i, j) \in \lambda} \tilde{G}^{\beta}\left(z_{\beta}(N+j-i)\right) .
$$

We may also include weight factors in which some or all of the parameters $\left(z_{1}, z_{2}, \ldots\right)$, $\left(w_{1}, w_{2}, \ldots\right)$ are repeated in the product. This only affects the constraints on the sums of the colengths in the weighted multispecies Hurwitz numbers. (See, e.g., Example 3.3 in [12], in which the weights are uniform, but the linear generating function that gives Hurwitz numbers for Belyi curves and strictly monotonic paths is replaced by a power of the latter, resulting in multiple branch points, with the total colength fixed, and multimononic paths.) 


\subsection{The multiparameter family of $\tau$-functions $\tau^{G^{(l, m)}(\mathrm{w}, \mathrm{z})}(\mathrm{t}, \mathrm{s})$}

The multispecies partitions are divided into two classes: those corresponding to the weight factors of type $G^{\alpha}(w)$, labelled $\left\{\mu^{\left(\alpha, u_{\alpha}\right)}\right\}_{1 \leq u_{\alpha} \leq k_{\alpha}}$ and those corresponding to dual type $\tilde{G}^{\beta}(z)$,

labelled $\left\{\nu^{\left(\beta, v_{\beta}\right.}\right\}_{1 \leq v_{\beta} \leq \tilde{k}_{\beta}}$. These are further subdivided into $l$ "colours", or "species" for the first class, denoted by the label $\alpha=1, \ldots, l$ and $m$ in the second, denoted by $\beta=1, \ldots, m$. Any given configuration $\left\{\left\{\mu^{\left(\alpha, u_{\alpha}\right)}\right\}_{1 \leq u_{\alpha} \leq k_{\alpha}},\left\{\nu^{\left(\beta, v_{\beta}\right.}\right\}_{1 \leq v_{\beta} \leq \tilde{k}_{\beta}}\right\}$ has $k_{\alpha}$ elements of colour $\alpha$ in the first class and $\tilde{k}_{\beta}$ elements of colour $\beta$ in the second class, for a total of

$$
k=\sum_{\alpha=1}^{l} k_{\alpha}+\sum_{\beta=1}^{m} \tilde{k}_{\beta}
$$

partitions.

Denoting the $l+m$ expansion parameters as

$$
\mathbf{w}=\left(w_{1}, \ldots, w_{l}\right), \quad \mathbf{z}=\left(z_{1}, \ldots, z_{m}\right),
$$

the multispecies weight generating function is formed from the product

$$
G^{(l, m)}(\mathbf{w}, \mathbf{z}):=\prod_{\alpha=1}^{l} G^{\alpha}\left(w_{\alpha}\right) \prod_{\beta=1}^{m} \tilde{G}^{\beta}\left(z_{\beta}\right),
$$

where each factor has an infinite product representation that is of one of the two types

$$
\begin{aligned}
& G^{\alpha}(w)=\prod_{i=1}^{\infty}\left(1+c_{i}^{\alpha} w\right), \quad \alpha=1, \ldots, l, \\
& \tilde{G}^{\beta}(w)=\prod_{i=1}^{\infty}\left(1-\tilde{c}_{i}^{\beta} w\right)^{-1}, \quad \beta=1, \ldots, m,
\end{aligned}
$$

for $l+m$ infinite sequences of parameters

$$
\mathbf{c}^{\alpha}=\left(c_{1}^{\alpha}, c_{2}^{\alpha}, \ldots\right), \quad \alpha=1, \ldots, l, \quad \tilde{\mathbf{c}}^{\beta}=\left(\tilde{c}_{1}^{\beta}, \tilde{c}_{2}^{\beta}, \ldots\right), \quad \beta=1, \ldots, m .
$$

Following the approach developed in [12], we define an associated element $G_{n}^{(l, m)}(\mathbf{w}, \mathbf{z}, \mathcal{J})$ of the center $\mathbf{Z}\left(\mathbf{C}\left[S_{n}\right]\right)$ of the group algebra $\mathbf{C}\left[S_{n}\right]$ by

$$
G_{n}^{(l, m)}(\mathbf{w}, \mathbf{z}, \mathcal{J}):=\prod_{a=1}^{n}\left(\prod_{\alpha=1}^{l} G^{\alpha}\left(w_{\alpha} \mathcal{J}_{a}\right)\right)\left(\prod_{\beta=1}^{m} \tilde{G}^{\beta}\left(z_{\beta} \mathcal{J}_{a}\right)\right),
$$

where $\mathcal{J}:=\left(\mathcal{J}_{1}, \ldots, \mathcal{J}_{n}\right)$ are the Jucys-Murphy elements (1.23) of $\mathbf{C}\left[S_{n}\right]$. The element $G_{n}^{(l, m)}(\mathbf{w}, \mathbf{z}, \mathcal{J})$ defines an endomorphism of $\mathbf{Z}\left(\mathbf{C}\left[S_{n}\right]\right)$ under multiplication that is diagonal in the basis $\left\{F_{\lambda}\right\}$ of $\mathbf{Z}\left(\mathbf{C}\left[S_{n}\right]\right)$ consisting of the orthogonal idempotents corresponding to irreducible representations, labelled by partitions $\lambda$ of $n$

$$
G_{n}^{(l, m)}(\mathbf{w}, \mathbf{z}, \mathcal{J}) F_{\lambda}=r_{\lambda}^{G^{(l, m)}(\mathbf{w}, \mathbf{z})} F_{\lambda} .
$$

The eigenvalues are

$$
r_{\lambda}^{G^{(l, m)}(\mathbf{w}, \mathbf{z})}=\prod_{\alpha=1}^{l} r_{\lambda}^{G^{\alpha}\left(w_{\alpha}\right)} \prod_{\beta=1}^{m} r_{\lambda}^{\tilde{G}^{\beta}\left(z_{\beta}\right)},
$$


where, as before,

$$
r_{\lambda}^{G^{\alpha}\left(w_{\alpha}\right)}:=\prod_{(i j) \in \lambda} G^{\alpha}\left(w_{\alpha}(j-i)\right), \quad r_{\lambda}^{\tilde{G}\left(z_{\beta}\right)}:=\prod_{(i j) \in \lambda} \tilde{G}^{\beta}\left(z_{\beta}(j-i)\right) .
$$

The associated hypergeometric $\tau$-function is

$$
\tau^{G^{(l, m)}(\mathbf{w}, \mathbf{z})}(\mathbf{t}, \mathbf{s})=\sum_{\lambda} r_{\lambda}^{G^{(l, m)}(\mathbf{w}, \mathbf{z})} s_{\lambda}(\mathbf{t}) s_{\lambda}(\mathbf{s})
$$

\subsection{Multispecies geometric weighted Hurwitz numbers}

The weight assigned to a multispecies $n$-sheeted branched covering of the Riemann sphere with a pair of branch points at, say, $(0, \infty)$ having ramification profiles $(\mu, \nu)$ and a further

$$
\sum_{\alpha=1}^{l} k_{\alpha}+\sum_{\beta=1}^{l} \tilde{k}_{\beta}
$$

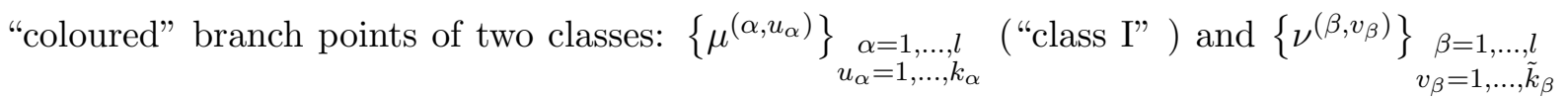
("class II") and $l+m$ colours $\{\alpha=1, \ldots, l\},\{\beta=1, \ldots, m\}$, with $\left\{k_{\alpha}\right\}_{\alpha=1, \ldots, l},\left\{\tilde{k}_{\beta}\right\}_{\beta=1, \ldots, m}$ points of the various colours (or "species"), is defined to be the product of those for single species

$$
W_{G^{(l, m)}}\left(\left\{\mu^{\left(\alpha, u_{\alpha}\right)}\right\},\left\{\nu^{\left(\beta, v_{\beta}\right.}\right\}\right)=\prod_{\alpha=1}^{l} m_{\lambda^{(\alpha)}}\left(\mathbf{c}^{(\alpha)}\right) \prod_{\beta=1}^{m} m_{\tilde{\lambda}^{(\beta)}}\left(\tilde{\mathbf{c}}^{\beta}\right) .
$$

Here the partitions $\left\{\lambda^{(\alpha)}\right\}_{\alpha=1, \ldots, l}$, and $\left\{\tilde{\lambda}_{\beta=1, \ldots, m}^{(\beta)}\right\}$ have parts equal to the colengths $\ell^{*}\left(\mu^{\left(\alpha, u_{\alpha}\right)}\right)$ and $\ell^{*}\left(\mu^{\left(\beta, v_{\beta}\right)}\right)$, for $\lambda^{(\alpha)}$ and $\tilde{\lambda}^{(\beta)}$ respectively. Their lengths are

$$
\ell\left(\lambda^{(\alpha)}\right)=k_{\alpha}, \quad \ell\left(\tilde{\lambda}^{(\beta)}\right)=\tilde{k}_{\beta}
$$

and their weights

$$
\left.\mid \lambda^{(\alpha)}\right)\left|=d_{\alpha}, \quad\right| \tilde{\lambda}^{(\beta)} \mid=\tilde{d}_{\beta}
$$

are equal to the specified total colengths

$$
\begin{aligned}
& \mathbf{d}=\left(d_{1}, \ldots, d_{l}\right), \quad \tilde{\mathbf{d}}=\left(d_{1}, \ldots, \tilde{d}_{m}\right), \\
& d_{\alpha}=\sum_{u_{\alpha}=1}^{k_{\alpha}} \ell^{*}\left(\mu^{\left(\alpha, u_{\alpha}\right)}\right), \quad \tilde{d}_{\beta}=\sum_{v_{\beta}=1}^{\tilde{k}_{\beta}} \ell^{*}\left(\nu^{\left(\beta v_{\beta}\right)}\right) .
\end{aligned}
$$

The geometrically defined multispecies weighted Hurwitz numbers are

$$
\begin{aligned}
H_{G^{(l, m)}}^{(\mathbf{d}, \tilde{\mathbf{d}})}(\mu, \nu):= & \sum_{k_{1}, \ldots, k_{l}} \sum_{\tilde{k}_{1}, \ldots, \tilde{k}_{m}} \sum_{\substack{\left\{\mu^{\left(\alpha, u_{\alpha}\right)}\right\} \\
\left|\mu^{\left(\alpha, u_{\alpha}\right)}\right|=n}}^{\prime} \sum_{\substack{\left\{\nu^{\left(\beta, v_{\beta}\right)}\right\} \\
\left|\nu^{\left(\beta, u_{\beta}\right)}\right|=n}}^{\prime} \sum_{\sum_{\alpha}=1}^{k_{\alpha} \ell^{*}\left(\mu^{\left(\alpha, u_{\alpha}\right)}\right)=d_{\alpha}} \sum_{v_{\beta}=1}^{\tilde{k}_{\beta} \ell^{*}\left(\nu^{\left(\beta, v_{\beta}\right)}\right)=\tilde{d}_{\beta}} \\
& \times W_{G^{(l, m)}}\left(\left\{\mu^{\left(\alpha, u_{\alpha}\right)}\right\},\left\{\nu^{\left(\beta, v_{\beta}\right)}\right\}\right) H\left(\left\{\mu^{\left(\alpha, u_{\alpha}\right)}\right\},\left\{\nu^{\left(\beta, v_{\beta}\right)}\right\}, \mu, \nu\right),
\end{aligned}
$$


which gives the weighted sum of the Hurwitz numbers of $n$-sheeted branched coverings with $l+m$ branch points of type $\left\{\left\{\mu^{\left(\alpha, u_{\alpha}\right)}\right\}_{1 \leq u_{\alpha} \leq k_{\alpha}},\left\{\nu^{\left(\beta, v_{\beta}\right.}\right\}_{1 \leq v_{\beta} \leq \tilde{k}_{\beta}}\right\}$ and $(\mu, \nu)$ at $(0, \infty)$,

Substituting the Frobenius-Schur formula (1.3) and the Frobenius character formula (1.31) into (2.12), it follows that $\tau^{G^{(l, m)}(\mathbf{w}, \mathbf{z})}(\mathbf{t}, \mathbf{s})$ is the generating function for $H_{G^{(l, m)}}^{(\mathbf{d}, \tilde{\mathbf{d}})}(\mu, \nu)$. Using multi-index notion to denote

$$
\prod_{\alpha=1}^{l} w_{\alpha}^{d_{\alpha}} \prod_{\beta=1}^{m} z_{\beta}^{\tilde{d}_{\beta}}=: \mathbf{w}^{\mathbf{d}} \mathbf{z}^{\tilde{\mathbf{d}}}
$$

we then have

\section{Theorem 2.1.}

$$
\tau^{G^{(l, m)}(\mathbf{w}, \mathbf{z})}(\mathbf{t}, \mathbf{s})=\sum_{\mathbf{d} \in \mathbf{N}} \mathbf{w}^{\mathbf{d}} \sum_{\tilde{\mathbf{d}} \in \mathbf{N}} \mathbf{z}^{\tilde{\mathbf{d}}} \sum_{\mu, \nu} H_{G^{(l, m)}}^{(\mathbf{d}, \tilde{\mathbf{d}})}(\mu, \nu) p_{\mu}(\mathbf{t}) p_{\nu}(\mathbf{s}) .
$$

Proof. This follows, as in the single species case, by combining the eigenvalue formula (2.9) with the Frobenius character formula.

\subsection{Multispecies combinatorial weighted Hurwitz numbers}

The combinatorial multispecies weighted Hurwitz number $F_{G^{(l, m)}}^{(\mathbf{d} \tilde{\mathbf{d}})}(\mu, \nu)$ is defined as follows. Let $D_{n}$ be the number of partitions of $n$ and let $\mathbf{F}_{G^{\alpha}}^{d_{\alpha}}$ and $\mathbf{F}_{\tilde{G}^{\beta}}^{\tilde{d}_{\beta}}$ denote the $D_{n} \times D_{n}$ matrices whose elements are $F_{G^{\alpha}}^{d_{\alpha}}(\mu, \nu)$ and $F_{\tilde{G}^{\beta}}^{\tilde{d}_{\beta}}(\mu, \nu)$, respectively, as defined in (1.19), (1.20),

$$
F_{G^{\alpha}}^{d}(\mu, \nu):=\frac{1}{n !} \sum_{\lambda} e_{\lambda}\left(\mathbf{c}^{\alpha}\right) m_{\mu \nu}^{\lambda}, \quad F_{\tilde{G}^{\beta}}^{d}(\mu, \nu):=\frac{1}{n !} \sum_{\lambda} h_{\lambda}\left(\tilde{\mathbf{c}}^{\beta}\right) m_{\mu \nu}^{\lambda},
$$

for each generating function $G^{\alpha}\left(w_{\alpha}\right)$ or $\tilde{G}^{\beta}\left(z_{\beta}\right)$. Since the central elements $\left\{G_{n}^{\alpha}\left(w_{\alpha}, \mathcal{J}\right)\right.$, $\left.\tilde{G}_{n}^{\beta}\left(z_{\beta}, \mathcal{J}\right)\right\}$ all commute, it follows that so do the matrices $\left\{\mathbf{F}_{G^{\alpha}}^{d_{\alpha}}, \mathbf{F}_{\tilde{G}^{\beta}}^{\tilde{d}_{\beta}}\right\}$. Denoting their product, in any order,

$$
\mathbf{F}_{G^{(l, m)}}^{(\mathbf{d}, \tilde{\mathbf{d}})}:=\prod_{\alpha=1}^{l} \mathbf{F}_{G^{\alpha}}^{d_{\alpha}} \prod_{\beta=1}^{m} \mathbf{F}_{\tilde{G}^{\beta}}^{\tilde{d}_{\beta}},
$$

the $(\mu, \nu)$ matrix element $F_{G^{(l, m)}}^{(\mathbf{d} \tilde{\mathbf{d}})}(\mu, \nu)$ is the combinatorial multispecies weighted Hurwitz number.

The combinatorial meaning of $F_{G^{(l, m)}}^{(\mathbf{d}, \tilde{\mathbf{d}})}(\mu, \nu)$ is as follows. Let

$$
d:=\sum_{\alpha=1}^{l} d_{\alpha}+\sum_{\beta=1}^{m} \tilde{d}_{\beta}
$$

Then $F_{G(l, m)}^{(\mathbf{d}, \tilde{\mathbf{d}})}(\mu, \nu)$, may be interpreted as the weighted sum over all sequences of $d$ step paths in the Cayley graph from an element $h \in \operatorname{cyc}(\mu)$ in the conjugacy class of cycle type $\mu$ to one $\left(a_{d} b_{d}\right) \cdots\left(a_{1} b_{1}\right) h \in \operatorname{cyc}(\nu)$ in the class cyc $(\nu)$, in which the transpositions appearing are subdivided into subsets consisting of $\left(d_{1}, \ldots, d_{l}, \tilde{d}_{1}, \ldots, \tilde{d}_{m}\right)$ transpositions in all $\frac{d !}{\prod_{\alpha=1}^{l} d_{\alpha} ! \prod_{\beta=1}^{l} \tilde{d}_{\beta} !}$ possible ways. All paths are divided into equivalence classes, according to their multisignatures 
$\left\{\lambda^{(\alpha)}, \tilde{\lambda}^{(\beta)}\right\}_{\substack{\alpha=1, \ldots, l \\ \beta=1, \ldots, m}}$. These consist of a partition of the $d$ steps into $l+m$ parts, each of which is a subsequence assigned a "colour" and a "class" with $l$ of them of the first class and $m$ of the second. The number of partitions of first class with colour $\alpha$ is $d_{\alpha}$ while the number of second class with colour $\beta$ is $\tilde{d}_{\beta}$. The partitions $\lambda^{(\alpha)}$ of weights $d_{\alpha}$ are defined to have parts $\left\{\lambda_{u_{\alpha}}^{(\alpha)}\right\}_{u_{\alpha}=1, \ldots, k_{\alpha}}$ equal to the number of transpositions appearing within that subsequence having the same second element, and similarly for $\left\{\tilde{\lambda}_{v_{\beta}}^{(\beta)}\right\}_{v_{\beta}=1, \ldots, \tilde{k}_{\beta}}$ with

$$
k_{\alpha}=\ell\left(\lambda^{(\alpha)}\right), \quad \tilde{k}_{\beta}=\ell\left(\tilde{\lambda}^{(\beta)}\right)
$$

the number of such parts.

The weight given to any such multisignatured path is the product

$$
\prod_{\alpha=1}^{l} e_{\lambda^{(\alpha)}}\left(\mathbf{c}^{\alpha}\right) \prod_{\beta=1}^{m} h_{\tilde{\lambda}^{(\beta)}}\left(\tilde{\mathbf{c}}^{\beta}\right)
$$

of the weights along each segment, and $F_{G^{(l, m)}}^{(\mathbf{d}, \tilde{\mathbf{d}})}(\mu, \nu)$ is the sum of these, each multiplied by the number of elements of the equivalence class of paths with the given multisignature.

The multispecies generalization of (1.21) is equality of the geometric and combinatorial Hurwitz numbers:

\section{Theorem 2.2.}

$$
F_{G^{(l, m)}}^{(\mathbf{d}, \tilde{\mathbf{d}})}(\mu, \nu)=H_{G(l, m)}^{(\mathbf{d}, \tilde{\mathbf{d}})}(\mu, \nu), \quad F_{\tilde{G}(l, m)}^{(\mathbf{d}, \tilde{\mathbf{d}})}(\mu, \nu)=H_{\tilde{G}(l, m)}^{(\mathbf{d}, \tilde{\mathbf{d}})}(\mu, \nu) .
$$

Proof. Applying the central element $G_{n}^{(l, m)}(\mathbf{w}, \mathbf{z}, \mathcal{J})$ defined in $(2.8)$ to the cycle sum $C_{\mu}$ and applying (1.26) for each factor in the product gives

$$
G_{n}^{(l, m)}(\mathbf{w}, \mathbf{z}, \mathcal{J}) C_{\mu}=\sum_{\nu,|\nu|=|\mu|} F_{G^{(l, m)}}^{(\mathbf{d}, \tilde{\mathbf{d}})}(\mu, \nu) C_{\nu}=\sum_{\nu,|\nu|=|\mu|} H_{G^{(l, m)}}^{(\mathbf{d}, \tilde{\mathbf{d}})}(\mu, \nu) C_{\nu},
$$

and the similar formula for $\tilde{G}$. Equation (2.21), together with the Frobenius character formula, shows that the generating $\tau$-function can be expressed as

$$
\tau^{G^{(l, m)}(\mathbf{w}, \mathbf{z})}(\mathbf{t}, \mathbf{s})=\sum_{\mathbf{d} \in \mathbf{N}} \mathbf{w}^{\mathbf{d}} \sum_{\tilde{\mathbf{d}} \in \mathbf{N}} \mathbf{z}^{\tilde{\mathbf{d}}} \sum_{\mu, \nu} F_{G^{(l, m)}}^{(\mathbf{d}, \tilde{\mathbf{d}})}(\mu, \nu) p_{\mu}(\mathbf{t}) p_{\nu}(\mathbf{s}) .
$$

Comparing this with equation (2.21) proves the result.

\section{Multispecies quantum Hurwitz numbers}

\subsection{Quantum Hurwitz numbers}

Amongst the examples of weighted Hurwitz numbers studied in [12], four special classes were introduced in which the generating functions $G(z), \tilde{G}(z)$ were chosen as a variant of the quantum dilogarithm [6]. This meant that the parameters $\left\{c_{i}\right\}$ were chosen as powers of a quantum deformation parameter $q$. As shown in [12], a suitable interpretation of the parameter $q$ in terms of Planck's constant $\hbar$ and Boltzmann factors for a Bosonic gas with linear energy spectrum leads to a relation between the resulting weighted counting of branched cover and the energy distribution for a Bosonic gas, which further justifies terming these "quantum" Hurwitz numbers. 
In the first case, the weight generating function is

$$
E(q, z):=\prod_{i=0}^{\infty}\left(1+q^{i} z\right)=1+\sum_{i=1}^{\infty} E_{i}(q) z^{i}, \quad E_{i}(q):=\left(\prod_{j=1}^{i} \frac{q^{j-1}}{1-q^{j}}\right)
$$

and hence the parameters $c_{i}$ are identified as $\left\{c_{i}:=q^{i-1}\right\}_{i \in \mathbf{N}^{+}}$. The second is a slight modification of this, with weight generating function

$$
E^{\prime}(q, z):=\prod_{i=1}^{\infty}\left(1+q^{i} z\right)=1+\sum_{i=1}^{\infty} E_{i}^{\prime}(q) z^{i}, \quad E_{i}^{\prime}(q):=\left(\prod_{j=1}^{i} \frac{q^{i}}{1-q^{j}}\right),
$$

i.e., the zero power $q^{0}$ is omitted, and $\left\{c_{i}:=q^{i}\right\}_{i \in \mathbf{N}^{+}}$.

The third case is based on the weight generating function

$$
H(q, z):=\prod_{i=0}^{\infty}\left(1-q^{i} z\right)^{-1}=1+\sum_{i=1}^{\infty} H_{i}(q) z^{i}, \quad H_{i}(q):=\left(\prod_{j=1}^{i} \frac{1}{1-q^{j}}\right),
$$

and hence is the dual of the first case, with $\left\{\tilde{c}_{i}:=q^{i-1}\right\}_{i \in \mathbf{N}^{+}}$. The final case is a hybrid, formed from the product of the first and third for two distinct quantum deformation parameters $q$ and $p$, with weight generating function

$$
\begin{aligned}
& Q(q, p, z):=\prod_{k=0}^{\infty}\left(1+q^{k} z\right)\left(1-p^{k} z\right)^{-1}=\sum_{i=0}^{\infty} Q_{i}(q, p) z^{i} \\
& Q_{i}(q, p):=\sum_{m=0}^{i} q^{\frac{1}{2} m(m-1)}\left(\prod_{j=1}^{m}\left(1-q^{j}\right) \prod_{j=1}^{i-m}\left(1-p^{j}\right)\right)^{-1}, \quad Q_{\lambda}(q, p)=\prod_{i=1}^{\ell(\lambda)} Q_{\lambda_{i}}(q, p) .
\end{aligned}
$$

These are all expressible as exponentials of the quantum dilogarithm function

$$
\begin{aligned}
& \operatorname{Li}_{2}(q, z):=\sum_{k=1}^{\infty} \frac{z^{k}}{k\left(1-q^{k}\right)}, \\
& E(q, z)=e^{-\operatorname{Li}_{2}(q,-z)}, \quad E^{\prime}(q, z)=(1+z)^{-1} e^{-\operatorname{Li}_{2}(q,-z)}, \\
& H(q, z)=e^{\operatorname{Li}_{2}(q, z)}, \quad Q(q, p, z)=e^{\mathrm{Li}_{2}(p, z)-\mathrm{Li}_{2}(q,-z)} .
\end{aligned}
$$

The content product formulae for the first and third of these are

$$
r_{\lambda}^{E(q, z)}(N):=\prod_{(i j) \in \lambda} E(q,(N+j-i) z), \quad r_{\lambda}^{H(q, z)}(N):=\prod_{(i j) \in \lambda} H(q,(N+j-i) z) .
$$

The associated hypergeometric $2 D$ Toda $\tau$-functions have diagonal double Schur function expansions with these as coefficients

$$
\begin{aligned}
\tau^{E(q, z)}(N, \mathbf{t}, \mathbf{s}) & =\sum_{\lambda} r_{\lambda}^{E(q, z)}(N) S_{\lambda}(\mathbf{t}) S_{\lambda}(\mathbf{s}), \\
\tau^{H(q, z)}(N, \mathbf{t}, \mathbf{s}) & \left.=\sum_{\lambda} r_{\lambda}^{H(q, z)}(N) S_{\lambda}(\mathbf{t}) S_{\lambda}(\mathbf{s})\right) .
\end{aligned}
$$

Using the Frobenius character formula (1.31), and setting $N=0$, they may be rewritten as double expansions in the power sum symmetric functions [12]

$$
\tau^{E(q, z)}(\mathbf{t}, \mathbf{s}):=\tau^{E(q, z)}(0, \mathbf{t}, \mathbf{s})=\sum_{d=0}^{\infty} z^{d} \sum_{\mu, \nu,|\mu|=|\nu|} H_{E(q)}^{d}(\mu, \nu) p_{\mu}(\mathbf{t}) p_{\nu}(\mathbf{s}),
$$




$$
\tau^{H(q, z)}(\mathbf{t}, \mathbf{s}):=\tau^{H(q, z)}(0, \mathbf{t}, \mathbf{s})=\sum_{d=0}^{\infty} z^{d} \sum_{\mu, \nu,|\mu|=|\nu|} H_{H(q)}^{d}(\mu, \nu) p_{\mu}(\mathbf{t}) p_{\nu}(\mathbf{s}) .
$$

The coefficients are the corresponding quantum Hurwitz numbers $H_{E(q)}^{d}(\mu, \nu), H_{H(q)}^{d}(\mu, \nu)$, which count weighted $n$-sheeted branched coverings of the Riemann sphere, defined by

$$
\begin{aligned}
& H_{E(q)}^{d}(\mu, \nu):=\sum_{k=0}^{\infty} \sum_{\substack{\mu^{(1)}, \ldots, \mu^{(k)} \\
\sum_{i=1}^{k} \ell^{*}\left(\mu^{(i)}\right)=d}}^{\prime} W_{E(q)}\left(\mu^{(1)}, \ldots, \mu^{(k)}\right) H\left(\mu^{(1)}, \ldots, \mu^{(k)}, \mu, \nu\right), \\
& H_{H(q)}^{d}(\mu, \nu):=\sum_{k=0}^{\infty}(-1)^{k+d} \sum_{\substack{\mu^{(1)}, \ldots, \mu^{(k)} \\
\sum_{i=1}^{k} \ell^{*}\left(\mu^{(i)}\right)=d}}^{\prime} W_{H(q)}\left(\mu^{(1)}, \ldots, \mu^{(k)}\right) H\left(\mu^{(1)}, \ldots, \mu^{(k)}, \mu, \nu\right),
\end{aligned}
$$

where the weightings for such covers with $k$ additional branch points are [12]

$$
\begin{aligned}
& W_{E(q)}\left(\mu^{(1)}, \ldots, \mu^{(k)}\right) \\
& =\frac{1}{|\operatorname{aut}(\lambda)|} \sum_{\sigma \in S_{k}} \frac{q^{(k-1) \ell^{*}\left(\mu^{(1)}\right)} \cdots q^{\ell^{*}\left(\mu^{(k-1)}\right)}}{\left(1-q^{\ell^{*}\left(\mu^{(\sigma(1))}\right)}\right) \cdots\left(1-q^{\ell^{*}\left(\mu^{(\sigma(1))}\right)}\right) \cdots q^{\ell^{*}\left(\mu^{(\sigma(k))}\right)},} \\
& W_{H(q)}\left(\mu^{(1)}, \ldots, \mu^{(k)}\right) \\
& =\frac{(-1)^{\ell^{*}(\lambda)}}{|\operatorname{aut}(\lambda)|} \sum_{\sigma \in S_{k}} \frac{1}{\left(1-q^{\ell^{*}\left(\mu^{(\sigma(1))}\right)}\right) \cdots\left(1-q^{\ell^{*}\left(\mu^{(\sigma(1))}\right)}\right) \cdots q^{\ell^{*}\left(\mu^{(\sigma(k))}\right)}} .
\end{aligned}
$$

Here, as in (1.8) and (1.14), $\lambda$ is the partition of length $\ell(\lambda)=k$ whose parts are $\left\{\ell^{*}\left(\mu^{(i)}\right)\right\}_{i=1, \ldots, k}$, and $|\operatorname{aut}(\lambda)|$ is the order of the automorphism group of $\lambda$. The $\operatorname{sum} \sum_{\mu^{(1)}, \ldots, \mu}^{\prime}(k)$ is over $\sum_{i=1}^{k} \ell^{*}\left(\mu^{(i)}\right)=d$

all $k$-tuples of partitions having nontrivial ramification profiles that satisfy the constraint $\sum_{i=1}^{k} \ell^{*}\left(\mu^{(i)}\right)=d$, and $H\left(\mu^{(1)}, \ldots, \mu^{(k)}, \mu, \nu\right)$ is the number of branched $n$-sheeted coverings, up to isomorphism, having $k+2$ branch points with ramification profiles $\left(\mu^{(1)}, \ldots, \mu^{(k)}, \mu, \nu\right)$.

These thus count weighted covers with a pair of branch points, say $(0, \infty)$, having ramification profiles of type $(\mu, \nu)$ and an arbitrary number of further branch points, whose profiles $\left(\mu^{(1)}\right.$, $\left.\ldots, \mu^{(k)}\right)$ are constrained only by the requirement that the sum of the colengths, which is related to the genus by the Riemann-Hurwitz formula

$$
\sum_{i=1}^{k} \ell^{*}\left(\mu^{(i)}\right)=2 g-2+\ell(\mu)+\ell(\nu)=d,
$$

be fixed to equal $d$.

The combinatorial interpretation of the quantum Hurwitz numbers $F_{E(q)}^{d}(\mu, \nu)$ and $F_{H(q)}^{d}(\mu, \nu)$ appearing in (3.13) is as follows. Let $\left(a_{1} b_{1}\right) \cdots\left(a_{d} b_{d}\right)$ be a product of $d$ transpositions $\left(a_{i} b_{i}\right) \in S_{n}$ in the symmetric group $S_{n}$ with $a_{i}<b_{i}, i=1, \ldots, d$. If $h \in S_{n}$ is in the conjugacy class $\operatorname{cyc}(\mu) \subset S_{n}$, we may view the successive steps in the product

$$
\left(a_{1} b_{1}\right) \cdots\left(a_{d} b_{d}\right) h
$$

as a path in the Cayley graph generated by all transpositions, whose signature is the partition $\lambda$ of $d,|\lambda|=d$, whose parts $\lambda_{i}$ consist of the number of transpositions $\left(a_{i} b_{i}\right)$ sharing the same 
second element. If we further require that the ones with equal second elements be grouped together into consecutive subsequences in which these second elements are constant, with the consecutive subsequences strictly increasing in their second elements, then the number $\tilde{N}_{\lambda}$ of elements with signature $\lambda$ is related to the number $N_{\lambda}$ of such ordered sequences by

$$
\tilde{N}_{\lambda}=\frac{|\lambda| !}{\prod_{i=1}^{\ell(\lambda)} \lambda_{i} !} N_{\lambda} .
$$

Denote the number of such paths from the conjugacy class of cycle type cyc $(\mu)$ to the one of type $\operatorname{cyc}(\nu)$ having signature $\lambda$ as $\tilde{m}_{\mu \nu}^{\lambda}$, and the number of ordered sequences of type $\lambda$ as $m_{\mu \nu}^{\lambda}$. Thus

$$
\tilde{m}_{\mu \nu}^{\lambda}=\frac{|\lambda| !}{\prod_{i=1}^{\ell(\lambda)} \lambda_{i} !} m_{\mu \nu}^{\lambda} .
$$

For all paths of signature $\lambda$ we assign the weights

$$
E_{\lambda}(q):=\prod_{i=1}^{\ell(\lambda)} E_{\lambda_{i}}(q)=\prod_{i=1}^{\ell(\lambda)} \frac{q^{\frac{1}{2} \lambda_{i}\left(\lambda_{i}-1\right)}}{\prod_{j=1}^{\lambda_{i}}\left(1-q^{j}\right)}, \quad H_{\lambda}(q):=\prod_{i=1}^{\ell(\lambda)} H_{\lambda_{i}}(q)=\prod_{i=1}^{\ell(\lambda)} \frac{1}{\prod_{j=1}^{\lambda_{i}}\left(1-q^{j}\right)}
$$

to paths in the Cayley graph, and obtain the pair of corresponding combinatorial weighted Hurwitz numbers

$$
\begin{aligned}
& F_{E(q)}^{d}(\mu, \nu):=\frac{1}{n !} \sum_{\lambda,|\lambda|=d} E_{\lambda}(q) m_{\mu \nu}^{\lambda}, \\
& F_{H(p)}^{d}(\mu, \nu):=\frac{1}{n !} \sum_{\lambda,|\lambda|=d} H_{\lambda}(q) m_{\mu \nu}^{\lambda},
\end{aligned}
$$

that give the weighted enumeration of paths, using the weighting factors $E_{\lambda}(q)$ and $H_{\lambda}(q)$ respectively for all paths of signature $\lambda$.

As shown in general in [12], the enumerative geometrical and combinatorial definitions of these quantum weighted Hurwitz numbers coincide:

$$
H_{E(q)}^{d}(\mu, \nu)=F_{E(q)}^{d}(\mu, \nu), \quad H_{H(q)}^{d}(\mu, \nu)=F_{H(q)}^{d}(\mu, \nu) .
$$

A similar result holds for weights generated by the function $E^{\prime}(q, z)$, with corresponding quantum Hurwitz numbers defined by

$$
H_{E^{\prime}(q)}^{d}(\mu, \nu):=\sum_{k=0}^{\infty} \sum_{\substack{\mu^{(1)}, \ldots, \mu^{(k)} \\ \sum_{i=1}^{k} \ell^{*}\left(\mu^{(i)}\right)=d}}^{\prime} W_{E^{\prime}(q)}\left(\mu^{(1)}, \ldots, \mu^{(k)}\right) H\left(\mu^{(1)}, \ldots, \mu^{(k)}, \mu, \nu\right),
$$

where the weights $W_{E^{\prime}(q)}\left(\mu^{(1)}, \ldots, \mu^{(k)}\right)$ are given by

$$
\begin{aligned}
& W_{E^{\prime}(q)}\left(\mu^{(1)}, \ldots, \mu^{(k)}\right):=\frac{1}{k !} \sum_{\sigma \in S_{k}} \frac{q^{(k) \ell^{*}\left(\mu^{(1)}\right)} \cdots q^{\ell^{*}\left(\mu^{(k)}\right)}}{\left(1-q^{\ell^{*}\left(\mu^{(\sigma(1))}\right)}\right) \cdots\left(1-q^{\ell^{*}\left(\mu^{(\sigma(1))}\right)}\right) \cdots q^{\ell^{*}\left(\mu^{(\sigma(k))}\right)}} \\
& :=\frac{1}{k !} \sum_{\sigma \in S_{k}} \frac{1}{\left(q^{-\ell^{*}\left(\mu^{(\sigma(1))}\right)}-1\right) \cdots\left(q^{-\ell^{*}\left(\mu^{(\sigma(1))}\right)} \cdots q^{-\ell^{*}\left(\mu^{(\sigma(k))}\right)}-1\right)} .
\end{aligned}
$$


Choosing $q$ as a positive real number, parametrizing it as

$$
q=e^{-\beta \hbar \omega_{0}}, \quad \beta=\frac{1}{k T}
$$

and identifying the energy levels $\epsilon_{k}$ as those for a Bose gas with linear spectrum in the integers, as for a $1-D$ harmonic oscillator

$$
\epsilon_{k}:=k \hbar \omega_{0}, \quad k \in \mathbf{N}
$$

it follows that if we assign the energy

$$
\epsilon(\mu):=\epsilon_{\ell^{*}(\mu)}=\ell^{*}(\mu) \hbar \omega
$$

to each branch point with ramification profile of type $\mu$, it contributes a factor

$$
n(\mu)=\frac{1}{e^{\beta \epsilon(\mu)}-1}
$$

to the weighting distributions, the same as that for a bosonic gas.

\subsection{The multiparameter family of $\tau$-functions $\tau^{Q(\mathrm{q} ; \mathrm{w} ; \mathrm{p}, \mathrm{z})}(N, \mathrm{t}, \mathrm{s})$}

We now consider the multiparameter family of weight generating functions $Q(\mathbf{q}, \mathbf{w} ; \mathbf{p}, \mathbf{z})$ obtained by taking the product of any number of the generating functions $E\left(q_{i}, z_{i}\right)$ and $H\left(p_{j}, w_{j}\right)$ for distinct sets of generating function parameters $\mathbf{w}=\left(w_{1}, \ldots, w_{l}\right), \mathbf{z}=\left(z_{1}, \ldots, z_{m}\right)$, and quantum parameters $\mathbf{q}=\left(q_{1}, \ldots, q_{l}\right), \mathbf{p}=\left(p_{1}, \ldots, p_{m}\right)$

$$
Q(\mathbf{q}, \mathbf{w} ; \mathbf{p}, \mathbf{z}):=\prod_{\alpha=1}^{l} E\left(q_{\alpha}, w_{\alpha}\right) \prod_{\beta=1}^{m} H\left(p_{\beta}, z_{\beta}\right) .
$$

Following the approach developed in [12], we define an associated element of the center $\mathbf{Z}\left(\mathbf{C}\left[S_{n}\right]\right)$ of the group algebra $\mathbf{C}\left[S_{n}\right]$ by

$$
Q_{n}(\mathbf{q}, \mathbf{w} ; \mathbf{p}, \mathbf{z}, \mathcal{J}):=\prod_{a=1}^{n} Q\left(\mathbf{q}, \mathcal{J}_{a} \mathbf{w} ; \mathbf{p}, \mathcal{J}_{a} \mathbf{z}\right),
$$

where $\mathcal{J}:=\left(\mathcal{J}_{1}, \ldots, \mathcal{J}_{n}\right)$ are again the Jucys-Murphy elements (1.23) of $\mathbf{C}\left[S_{n}\right]$. The element $Q_{n}(\mathbf{q}, \mathbf{w} ; \mathbf{p}, \mathbf{z}, \mathcal{J})$ defines an endomorphism of $\mathbf{Z}\left(\mathbf{C}\left[S_{n}\right]\right)$ under multiplication, which is diagonal in the basis $\left\{F_{\lambda}\right\}$ of $\mathbf{Z}\left(\mathbf{C}\left[S_{n}\right]\right)$ consisting of the orthogonal idempotents, corresponding to irreducible representations, labelled by partitions $\lambda$ of $n$

$$
Q_{n}(\mathbf{q}, \mathbf{w} ; \mathbf{p}, \mathbf{z}, \mathcal{J}) F_{\lambda}=r_{\lambda}^{Q(\mathbf{q}, \mathbf{w} ; \mathbf{p}, \mathbf{z})} F_{\lambda}
$$

where

$$
r_{\lambda}^{Q(\mathbf{q}, \mathbf{w} ; \mathbf{p}, \mathbf{z})}=\prod_{\alpha=1}^{l} r_{\lambda}^{E\left(q_{\alpha}\right)}\left(w_{\alpha}\right) \prod_{\beta=1}^{m} r_{\lambda}^{H\left(p_{\beta}\right)}\left(z_{\beta}\right) .
$$

More generally, defining

$$
r_{\lambda}^{Q(\mathbf{q}, \mathbf{w} ; \mathbf{p}, \mathbf{z})}(N)=\prod_{\alpha=1}^{l} r_{\lambda}^{E\left(q_{\alpha}, w_{\alpha}\right)}(N) \prod_{\beta=1}^{m} r_{\lambda}^{H\left(p_{\beta}, z_{\beta}\right)}(N),
$$


where

$$
\begin{aligned}
& r_{\lambda}^{E\left(q_{\alpha}, w_{\alpha}\right)}(N):=\prod_{(i j) \in \lambda} E\left(q_{\alpha},(N+j-i) w_{\alpha}\right), \\
& r_{\lambda}^{H\left(p_{\beta}, z_{\beta}\right)}(N):=\prod_{(i j) \in \lambda} H\left(p_{\beta},(N+j-i) z_{\beta}\right),
\end{aligned}
$$

we have

$$
r_{\lambda}^{Q(\mathbf{q}, \mathbf{z} ; \mathbf{p}, \mathbf{w})}=r_{\lambda}^{Q(\mathbf{q}, \mathbf{z} ; \mathbf{p}, \mathbf{w})}(0) .
$$

The double Schur function series

$$
\tau^{Q(\mathbf{q}, \mathbf{z} ; \mathbf{p}, \mathbf{w})}(N, \mathbf{t}, \mathbf{s}):=\sum_{\lambda} r_{\lambda}^{Q(\mathbf{q}, \mathbf{z} ; \mathbf{p}, \mathbf{w})}(N) S_{\lambda}(\mathbf{t}) S_{\lambda}(\mathbf{s})
$$

then defines a family of $2 D$ Toda $\tau$-functions of hypergeometric type.

\subsection{Multispecies geometric quantum Hurwitz numbers}

We now consider coverings in which the branch points are divided, as above, into two different classes, $\left\{\mu^{\left(\alpha, u_{\alpha}\right)}\right\}_{\alpha=1, \ldots, l ; u_{\alpha}=1, \ldots, k_{\alpha}}$ and $\left\{\nu^{\left(\beta, v_{\beta}\right)}\right\}_{\beta=1, \ldots, m ; v_{\beta}=1, \ldots, \tilde{k}_{\beta}}$, corresponding to weight generating functions of type $E\left(q_{\alpha}\right)$ and $H\left(p_{\beta}\right)$ respectively, each of which is further divided into $l$ and $m$ distinct species (or "colours"), of which there are $\left\{k^{\alpha}\right\}$ and $\left\{\tilde{k}^{\beta}\right\}$ branch points of types $E$ and $H$ and colours $\alpha$ and $\beta$ respectively. The weighted number of such coverings with specified total colengths $\mathbf{d}=\left(d_{1}, \ldots, d_{l}\right), \tilde{\mathbf{d}}=\left(d_{1}, \ldots, \tilde{d}_{m}\right), d_{\alpha}, \tilde{d}_{\beta} \in \mathbf{N}$

$$
d_{\alpha}=\sum_{u_{\alpha}=1}^{k_{\alpha}} \ell^{*}\left(\mu^{\left(\alpha, u_{\alpha}\right)}\right), \quad \tilde{d}_{\beta}=\sum_{v_{\beta}=1}^{\tilde{k}_{\beta}} \ell^{*}\left(\nu^{\left(\beta v_{\beta}\right)}\right)
$$

for each class and colour is the multispecies quantum Hurwitz number

$$
\begin{aligned}
& H_{(\mathbf{q}, \mathbf{p})}^{(\mathbf{d}, \tilde{\mathbf{d}})}(\mu, \nu):=\sum_{\substack{\left\{k_{\alpha}=1 ; \tilde{k}_{\beta}=1\right\} \\
\alpha=1, \ldots, j ; \beta=1, \ldots, m}}^{\infty} \sum_{\left\{\mu^{\left(\alpha, u_{\alpha}\right) ; \nu}\left(\beta, v_{\beta}\right)\right\}} \\
& \sum_{u_{\alpha}=1}^{k_{\alpha}} \ell^{*}\left({ }_{\mu}\left(\alpha, u_{\alpha}\right)\right)=d_{\alpha}, \sum_{v_{\beta}=1}^{\tilde{k}_{\beta}} \ell^{*}\left(\nu^{\left(\beta, v_{\beta}\right)}\right)=\tilde{d}_{\beta} \\
& \times \prod_{\alpha=1}^{l} W_{E\left(q_{\alpha}\right)}\left(\mu^{(\alpha, 1)}, \ldots, \mu^{\left(\alpha, k_{\alpha}\right)}\right) \\
& \times \prod_{\beta=1}^{m} W_{H\left(p_{\beta}\right)}\left(\nu^{(\beta, 1)}, \ldots, \nu^{\left(\beta, \tilde{k}_{\beta}\right)}\right) H\left(\left\{\mu^{\left(\alpha, u_{\alpha}\right)} ; \nu^{\left(\beta, v_{\beta}\right)}\right\}, \mu, \nu\right) \text {. }
\end{aligned}
$$

Substituting the Frobenius-Schur formula (1.3) and the Frobenius character formula (1.31) into (3.40), it follows that

$$
\tau_{Q(\mathbf{q}, \mathbf{z} ; \mathbf{p}, \mathbf{w})}(\mathbf{t}, \mathbf{s}):=\tau_{Q(\mathbf{q}, \mathbf{z} ; \mathbf{p}, \mathbf{w})}(0, \mathbf{t}, \mathbf{s})
$$

is the generating function for $H_{(\mathbf{q}, \mathbf{p})}^{(\mathbf{c}, \mathbf{d})}(\mu, \nu)$. Using multi-index notion to denote

$$
\prod_{\alpha=1}^{l} w_{\alpha}^{d_{\alpha}} \prod_{\beta=1}^{m} z_{\beta}^{\tilde{d}_{\beta}}=: \mathbf{w}^{\mathbf{d}} \mathbf{z}^{\tilde{\mathbf{d}}}
$$

we have 


\section{Theorem 3.1.}

$$
\tau^{Q(\mathbf{q}, \mathbf{w} ; \mathbf{p}, \mathbf{z})}(\mathbf{t}, \mathbf{s}):=\sum_{\mathbf{d} \in \mathbf{N}} \mathbf{w}^{\mathbf{d}} \sum_{\tilde{\mathbf{d}} \in \mathbf{N}} \mathbf{z}^{\tilde{\mathbf{d}}} \sum_{\mu, \nu} H_{(\mathbf{q}, \mathbf{p})}^{(\mathbf{d}, \tilde{\mathbf{d}})}(\mu, \nu) p_{\mu}(\mathbf{t}) p_{\nu}(\mathbf{s}) .
$$

\subsection{Multispecies combinatorial quantum Hurwitz numbers}

Another basis for $\mathbf{Z}\left(\mathbf{C}\left[S_{n}\right]\right)$ consists of the cycle sums

$$
C_{\mu}:=\sum_{h \in \operatorname{cyc}(\mu)} h
$$

where $\operatorname{cyc}(\mu)$ denotes the conjugacy class of elements $h \in \operatorname{cyc}(\mu)$ with cycle lengths equal to the parts of $\mu$. The two are related by

$$
F_{\lambda}=h_{\lambda}^{-1} \sum_{\mu,|\mu|=|\lambda|} \chi_{\lambda}(\mu) C_{\mu}
$$

where $\chi_{\lambda}(\mu)$ denotes the irreducible character of the irreducible representation of type $\lambda$ evaluated on the conjugacy class $\operatorname{cyc}(\mu)$. Under the characteristic map, this is equivalent to the Frobenius character formula (1.31).

Let $D_{n}$ denote the number of partitions of $n$ and $\mathbf{F}_{E\left(q_{\alpha}\right)}^{\left(n, d_{\alpha}\right)}, \mathbf{F}_{H\left(p_{\beta}\right)}^{\left(n, \tilde{d}_{\beta}\right)}$ the $D_{n} \times D_{n}$ matrices whose elements are $\left(F_{E\left(q_{\alpha}\right)}^{d_{\alpha}}(\mu, \nu)\right)_{|\mu|=|\nu|=n}$ and $\left(F_{H\left(p_{\beta}\right)}^{\tilde{d}_{\beta}}(\mu, \nu)\right)_{|\mu|=|\nu|=n}$, respectively, for $\alpha=1, \ldots, l$, $\beta=1, \ldots, m$ as defined in (3.23), (3.24). Since these represent central elements of the group algebra $\mathbf{C}\left[S_{n}\right]$, they commute amongst themselves. Defining the matrix product

$$
\mathbf{F}_{(\mathbf{q}, \mathbf{p})}^{(\mathbf{d}, \tilde{\mathbf{d}})}=\prod_{\alpha=1}^{l} \mathbf{F}_{E\left(q_{i}\right)}^{\left(n, d_{\alpha}\right)} \prod_{\beta=1}^{m} \mathbf{F}_{H\left(p_{\beta}\right)}^{\left(n, \tilde{d}_{\beta}\right)},
$$

its matrix elements, denoted $F_{(\mathbf{q}, \mathbf{p})}^{(\mathbf{c}, \mathbf{d})}(\mu, \nu)$, may be interpreted as the weighted number of

$$
d:=\sum_{\alpha=1}^{\alpha} d_{\alpha}+\sum_{\beta=1}^{m} \tilde{d}_{\beta}
$$

step paths in the Cayley graph from the conjugacy class of cycle type $\mu$ to the one of type $\nu$, where all paths are divided into equivalence classes, according to their multisignatures $\left\{\lambda^{(\alpha)}, \tilde{\lambda}^{(\beta)}\right\}_{\substack{\alpha=1, \ldots, l \\ \beta=1, \ldots, m}}$.

These consist of a partition of the $d$ steps into $l+m$ parts, each of which is a subsequence assigned a "colour" and a "class" with $l$ of them of the first class and $m$ of the second. The number of partitions of first class with colour $\alpha$ is $d_{\alpha}$ while the number of second class with colour $\beta$ is $\tilde{d}_{\beta}$. The partitions $\lambda^{(\alpha)}$ of weights $d_{\alpha}$ are defined to have parts $\left\{\lambda_{u_{\alpha}}^{(\alpha)}\right\}_{u_{\alpha}=1, \ldots, k_{\alpha}}$ equal to the number of a transposition appears within that subsequence, having the same second element, and similarly for $\left\{\tilde{\lambda}_{v_{\beta}}^{(\beta)}\right\}_{v_{\beta}=1, \ldots, \tilde{k}_{\beta}}$ with

$$
k_{\alpha}=\ell\left(\lambda^{(\alpha)}\right), \quad \tilde{k}_{\beta}=\ell\left(\tilde{\lambda}^{(\beta)}\right)
$$

the number of such parts.

For each such subpartition, the weight assigned is the product of the weights for each subsegment

$$
\prod_{\alpha=1}^{l} E_{\lambda^{(\alpha)}}\left(q_{\alpha}, w_{\alpha}\right) \prod_{\beta=1}^{m} H_{(\tilde{\lambda})^{\beta}}\left(p_{\beta}, z_{\beta}\right)
$$


and $F_{(\mathbf{q}, \mathbf{p})}^{(\mathbf{c}, \mathbf{d})}(\mu, \nu)$ is the sum of these, each multiplied by the number of elements of the equivalence class of paths with the given multisignature

$$
\begin{aligned}
F_{(\mathbf{q}, \mathbf{p})}^{(\mathbf{c}, \mathbf{d})}(\mu, \nu)= & (n !)^{-l-m} \sum_{\mu^{(2)}, \ldots, \mu^{(m+l)}} \prod_{\alpha=1}^{l}\left(\sum_{\left\{\lambda^{(\alpha)}\right\}} E_{\lambda^{(\alpha)}}\left(q_{\alpha}, w_{\alpha}\right) m_{\mu_{\alpha} \mu_{\alpha+1}}^{\lambda^{(\alpha)}}\right) \\
& \times \prod_{\beta=1}^{m}\left(\sum_{\left\{\tilde{\lambda}^{(\beta)}\right\}} H_{\tilde{\lambda}^{(\beta)}}\left(p_{\beta}, z_{\beta}\right) m_{\mu_{\beta+l}^{\lambda^{(\beta)}, \mu_{\beta+l+1}}}\right),
\end{aligned}
$$

where $(\mu, \nu):=\left(\mu^{(1)}, \mu^{(l+m+1)}\right)$.

The multispecies generalization of (3.25) is equality of the geometric and combinatorial Hurwitz numbers:

\section{Theorem 3.2.}

$$
F_{(\mathbf{q}, \mathbf{p})}^{(\mathbf{c}, \mathbf{d})}(\mu, \nu)=H_{(\mathbf{q}, \mathbf{p})}^{(\mathbf{c}, \mathbf{d})}(\mu, \nu) \text {. }
$$

Proof. Applying the central element (3.34) to the cycle sum $C_{\mu}$ gives

$$
\hat{Q}(\mathbf{q}, \mathbf{z} ; \mathbf{p}, \mathbf{w}, \mathcal{J}) C_{\mu}=\sum_{\nu,|\nu|=|\mu|} F_{(\mathbf{q}, \mathbf{p})}^{(\mathbf{c}, \mathbf{d})}(\mu, \nu) C_{\nu}
$$

and the orthogonality of group characters implies that

$$
\tau^{Q(\mathbf{q}, \mathbf{z} ; \mathbf{p}, \mathbf{w})}(\mathbf{t}, \mathbf{s}):=\sum_{\mathbf{c}=(0, \ldots, 0) ; \mathbf{d}=(0, \ldots, 0)}^{(\infty, \ldots, \infty)} \mathbf{z}^{\mathbf{c}} \mathbf{w}^{\mathbf{d}} \sum_{\mu, \nu} F_{(\mathbf{q}, \mathbf{p})}^{(\mathbf{c}, \mathbf{d})}(\mu, \nu) p_{\mu}(\mathbf{t}) p_{\nu}(\mathbf{s}) .
$$

Comparing this with equation (3.45) proves the result.

Remark 3.3 (multispecies Bose gases). Returning to the interpretation of the quantum Hurwitz weighting distributions in terms of Bose gases, if we choose each $q_{i}$ to be a positive real number with $q_{i}<1$, and parametrize it, as before,

$$
q_{i}=e^{-\beta \hbar \omega_{i}}
$$

for some ground state energy $\hbar \omega_{i}$, and again choose a linear energy spectrum, with energy assigned to the branchpoint $\mu$ of type $i$ with profile type $\mu$ to be

$$
\epsilon^{(i)}(\mu):=\ell^{*}(\mu) \hbar \omega_{i}
$$

we see that the resulting contributions to the weighting distributions distributions from each species of branch points of ramification type $\mu^{(j)}$ are given by

$$
n_{B}^{(i)}(\mu)=\frac{1}{e^{\beta \epsilon^{(i)}(\mu)}-1},
$$

which are those of a multispecies mixture of Bose gases.

\section{Acknowledgments}

This work is an extension of a joint project $[11,12]$ with M. Guay-Paquet, in which the notion of infinite parametric families of weighted Hurwitz numbers was first introduced, combined with the notion of signed multispecies Hurwitz numbers as introduced in [15] with A.Yu. Orlov. The author would like to thank both these co-authors for helpful discussions. Work supported by the Natural Sciences and Engineering Research Council of Canada (NSERC) and the Fonds de recherche du Québec - Nature et technologies (FRQNT). 


\section{References}

[1] Bouchard V., Mariño M., Hurwitz numbers, matrix models and enumerative geometry, in From Hodge Theory to Integrability and TQFT: tt*-Geometry, Proc. Sympos. Pure Math., Vol. 78, Amer. Math. Soc., Providence, RI, 2008, 263-283, arXiv:0709.1458.

[2] Diaconis P., Greene C., Applications of Murphy's elements, Stanford Technical Report, no. 335, 1989.

[3] Eynard B., Invariants of spectral curves and intersection theory of moduli spaces of complex curves, Commun. Number Theory Phys. 8 (2014), 541-588, arXiv:1110.2949.

[4] Eynard B., Orantin N., Invariants of algebraic curves and topological expansion, Commun. Number Theory Phys. 1 (2007), 347-452, math-ph/0702045.

[5] Eynard B., Orantin N., Topological recursion in enumerative geometry and random matrices, J. Phys. A: Math. Theor. 42 (2009), 293001, 117 pages, arXiv:0811.3531.

[6] Faddeev L.D., Kashaev R.M., Quantum dilogarithm, Modern Phys. Lett. A 9 (1994), 427-434, hep-th/9310070.

[7] Frobenius G., Über die Charaktere der symmetrischen Gruppe, Sitzungsber. Königl. Preuss. Akad. Wiss. (1900), 516-534.

[8] Frobenius G., Über die charakterische Einheiten der symmetrischen Gruppe, Sitzungsber. Königl. Preuss. Akad. Wiss. (1903), 328-358.

[9] Goulden I.P., Jackson D.M., The KP hierarchy, branched covers, and triangulations, Adv. Math. 219 (2008), 932-951, arXiv:0803.3980.

[10] Gross K.I., Richards D.S.P., Special functions of matrix argument. I. Algebraic induction, zonal polynomials, and hypergeometric functions, Trans. Amer. Math. Soc. 301 (1987), 781-811.

[11] Guay-Paquet M., Harnad J., 2D Toda $\tau$-functions as combinatorial generating functions, Lett. Math. Phys. 105 (2015), 827-852.

[12] Guay-Paquet M., Harnad J., Generating functions for weighted Hurwitz numbers, arXiv:1408.6766.

[13] Harnad J., Quantum Hurwitz numbers and Macdonald polynomials, arXiv:1504.03311.

[14] Harnad J., Weighted Hurwitz numbers and hypergeometric $\tau$-functions: an overview, arXiv:1504.03408.

[15] Harnad J., Orlov A.Yu., Hypergeometric $\tau$-functions, Hurwitz numbers and enumeration of paths, Comm. Math. Phys. 338 (2015), 267-284, arXiv:1407.7800.

[16] Hurwitz A., Ueber Riemann'sche Fläsche mit gegebnise Verzweigungspunkten, Math. Ann. 39 (1891), 1-60.

[17] Hurwitz A., Ueber die Anzahl der Riemann'sche Fläsche mit gegebnise Verzweigungspunkten, Math. Ann. 55 (1902), 53-66.

[18] Jucys A.-A.A., Symmetric polynomials and the center of the symmetric group ring, Rep. Math. Phys. 5 (1974), 107-112.

[19] Lando S.K., Zvonkin A.K., Graphs on surfaces and their applications, Encyclopaedia of Mathematical Sciences, Vol. 141, Springer-Verlag, Berlin, 2004.

[20] Macdonald I.G., Symmetric functions and Hall polynomials, 2nd ed., Oxford Mathematical Monographs, Oxford Science Publications, The Clarendon Press, Oxford University Press, New York, 1995.

[21] Murphy G.E., A new construction of Young's seminormal representation of the symmetric groups, J. Algebra 69 (1981), 287-297.

[22] Natanzon S.M., Orlov A.Yu., Hurwitz numbers and BKP hierarchy, arXiv:1407.8323.

[23] Natanzon S.M., Orlov A.Yu., BKP and projective Hurwitz numbers, arXiv:1501.01283.

[24] Okounkov A., Toda equations for Hurwitz numbers, Math. Res. Lett. 7 (2000), 447-453, math.AG/0004128.

[25] Orlov A.Yu., Shcherbin D.M., Hypergeometric solutions of soliton equations, Theoret. and Math. Phys. 128 (2001), 906-926.

[26] Pandharipande R., The Toda equations and the Gromov-Witten theory of the Riemann sphere, Lett. Math. Phys. 53 (2000), 59-74, math.AG/9912166.

[27] Schur I., Neue Begründung der Theorie der Gruppencharaktere, Sitzungsber. Königl. Preuss. Akad. Wiss. (1904), 406-432.

[28] Takasaki K., Initial value problem for the Toda lattice hierarchy, in Group Representations and Systems of Differential Equations (Tokyo, 1982), Adv. Stud. Pure Math., Vol. 4, North-Holland, Amsterdam, 1984, 139-163.

[29] Takebe T., Representation theoretical meaning of the initial value problem for the Toda lattice hierarchy. I, Lett. Math. Phys. 21 (1991), 77-84.

[30] Ueno K., Takasaki K., Toda lattice hierarchy, in Group Representations and Systems of Differential Equations (Tokyo, 1982), Adv. Stud. Pure Math., Vol. 4, North-Holland, Amsterdam, 1984, 1-95. 